\title{
Fisiología de la ventilación mecánica no invasiva
}

\section{Physiology of non-invasive mechanical ventilation}

\author{
Fabio Andrés Varón V., MD. ${ }^{(1)}$; Ángela María Giraldo M., MD. ${ }^{(2)}$
}

\section{Resumen}

La ventilación mecánica no invasiva se refiere a la entrega de ventilación a los pulmones utilizando técnicas que no requieren una vía aérea endotraqueal. La aplicación de presión positiva en ventilación no invasiva se remonta a la década de 1930, cuando los estudios de Barach demostraron que la presión positiva continua en la vía aérea, podría ser útil en el tratamiento del edema pulmonar agudo. Pero solo hasta 1980 se desarrollaron investigaciones administrando presión positiva a través de una boquilla con lo cual se obtuvieron resultados positivos con descenso en los niveles de $\mathrm{PaCO}_{2}$ y mejoría de la oxigenación en pacientes con EPOC y ASMA, sin embargo los resultados no fueron homogéneos por lo que se desestímulo su uso. Hoy se conoce que esta disparidad en los resultados se debió al corto tiempo de la ventilación mecánica no invasiva, de tan sólo 10 a 15 minutos tres o cuatro veces al día, demasiado breve para obtener buenos resultados. En paralelo, esta se utilizó en pacientes con enfermedad neuromuscular en el Centro de Rehabilitación de Goldwater en Nueva York, pero el uso de diferentes interfaces hizo que la técnica no presentara una adecuada adaptación en el grupo de pacientes (2). Sólo hasta 1985 se produjo una proliferación de su uso con la introducción de presión positiva continua en la vía aérea para el tratamiento de la apnea obstructiva del sueño. Hoy se han confirmado los beneficios en diversas entidades clínicas (3).

Palabras clave: ventilación no invasiva, trabajo muscular, constantes de tiempo, capacidad residual funcional, PEEP.

\begin{abstract}
Non-invasive mechanical ventilation (NIMV) refers to delivering ventilation to the lungs by means of techniques that do not require endotracheal intubation. Application of positive pressure by non-invasive ventilation dates back to the 1930s, when the studies of Alvan Barach showed that continuous positive airway pressure could be useful in the treatment of acute pulmonary edema (1). But it was not until 1980 that investigations were carried out by applying positive pressure via a mouthpiece, which achieved positive results by decreasing levels of $\mathrm{PaCO}_{2}$ and improving oxygenation in patients with COPD and asthma. However, since the results were not homogeneous, its use was discouraged. We now know that this disparity in the results was due to short time of NIMV, which was only 10-15 minutes, three or four times a day; this was too short to give good results. NIMV was at the same time used in patients with neuromuscular disease at the Goldwater Rehabilitation Center in New York, but the use of different interfaces made it impossible for this technique to adapt to the group of patients (2). Not until 1985 did its use become widespread, with application of continuous positive airway pressure (CPAP) for the treatment of obstructive sleep apnea. Today, its benefits have been confirmed for diverse clinical conditions (3).
\end{abstract}

Keywords: non-invasive ventilation, muscular work, time constants, functional residual capacity, PEEP.

\footnotetext{
(1) Jefe Unidad de Cuidado Intensivo y Programa de Trasplante Pulmonar, Fundación Neumológica Colombiana, Bogotá, Colombia.

${ }^{(2)}$ Fellow de Neumología, Fundación Neumológica Colombiana-Universidad de La Sabana. Bogotá, Colombia.

Correspondencia: Fabio Andrés Varón V., correo electrónico: fvaron@neumologica. org

Recibido: 10/03/16. Aceptado: 10/03/16.
} 


\section{Introducción}

La intubación orotraqueal ha sido el método de elección para la ventilación mecánica. No obstante, existe un aumento importante en el uso de la ventilación no invasiva generado por el deseo de reducir complicaciones de la ventilación invasiva y mejorar la utilización de los recursos. La ventilación no invasiva ha demostrado tener impacto positivo sobre la morbilidad hospitalaria, facilidad en el proceso del retiro de la ventilación mecánica, disminución del tiempo de hospitalización y en los costos, así como mayor comodidad para el paciente (4). Sin embargo, los casos deben ser seleccionados cuidadosamente, de acuerdo con criterios y conocimiento profundo de los mecanismos fisiológicos de efectividad de la ventilación mecánica no invasiva (VMNI) y de la condición a tratar, puesto que el riesgo de complicaciones podría aumentar si se utiliza de manera inapropiada.

Los objetivos de la VMNI son los mismos que que se persiguen con la ventilación mecánica invasiva: garantizar la adaptación y normalizar el intercambio gaseoso y minimizar el trabajo respiratorio del paciente. En casos con enfermedad cardiopulmonar o neurológica, la VMNI mejora el intercambio gaseoso a través del aumento del volumen corriente $(\mathrm{Vt})$ y garantiza así una ventilación alveolar adecuada (5). Al mejorar el Vt y utilizar la presión positiva al final de la espiración (PEEP), se puede mejorar la distensibilidad del sistema respiratorio debido al reclutamiento y a la estabilización de los alvéolos colapsados mejorando la aireación alveolar (6). Además, el aumento del Vt reduce la intensidad y duración de las contracciones de los músculos inspiratorios, por lo cual disminuyen el trabajo muscular (W) $(7,8)$. Sin embargo, el paciente bajo VMNI está sujeto a efectos potencialmente nocivos, como reducción del retorno venoso y del gasto cardíaco e hipotensión sistémica $(9,10)$.

El objetivo de este artículo es precisar el conocimiento acerca de los efectos fisiológicos de la VMNI respecto al trabajo respiratorio, el patrón respiratorio, la mecánica del sistema respiratorio, la función hemodinámica y el intercambio gaseoso.
En comienzo se aclararán algunos conceptos sobre las propiedades físicas del tejido pulmonar.

\section{Elasticidad, propiedades resistivas, trabajo respiratorio y difusión}

\section{Elasticidad}

Bajo un rango limitado de volúmenes, el pulmón reacciona como un resorte con determinadas presiones de distensión. Sin embargo, al llegar a cierto punto de expansión, establecido en la curva presión-volumen como el punto de inflexión superior, se activan mecanismos que limitan este rango (el menor nivel de inflación). En el punto de inflexión inferior se permite un incremento lineal de presiones para distender el tejido pulmonar colapsado. El retroceso elástico en el pulmón es causado por fibras elásticas propias del pulmón y por la fuerza generada por la tensión superficial de la interfase aire - líquido del alvéolo (11). Los mecanismos independientes evitan el colapso alveolar: la tensión superficial cuyo valor es independiente del diámetro del alvéolo y el fenómeno de interdependencia alveolar (hace que si un alvéolo pierde volumen, por fenómenos de tracción sobre los alvéolos adyacentes, se produzca homogenización y distribución uniforme del aire inspirado y estabilización posterior del tamaño alveolar).

\section{Propiedades resistivas}

La resistencia, además de la presión generada para distender el tejido pulmonar, necesita de un flujo de aire adicional (12). La resistencia es inversamente proporcional al diámetro de la vía aérea por lo que las divisiones bronquiales incrementarán de manera progresiva su contribución a la resistencia total (13). Los pacientes con enfermedad pulmonar obstructiva crónica (EPOC) durante la respiración espontánea tienden a respirar con mayores volúmenes por un incremento de la capacidad funcional residual (CFR) (definida como el punto de equilibrio entre la estabilidad de la caja torácica y el retroceso elástico del pulmón) y el aumento del volumen residual con tendencia a respirar hacia la capacidad pulmonar total. Si el pulmón está más insuflado se requieren mayores variaciones en la presión pleural 
y mayor trabajo de los músculos inspiratorios (W) para lograr distender el tejido pulmonar. Además, se activan fuerzas viscoelásticas del tejido pulmonar adyacente que incrementan la resistencia o el retroceso elástico.

\section{Constantes de tiempo}

El equilibrio entre el sistema de retroceso elástico, la resistencia y el final de la espiración determinan el tiempo necesario para el vaciamiento pulmonar, este tiempo es llamado constante de tiempo. Una constante de tiempo es el tiempo necesario para desocupar el 63\% del volumen alveolar. En un pulmón sano, aproximadamente después de tres constantes de tiempo, el volumen corriente puede ser espirado.

\section{Trabajo respiratorio}

La definición de trabajo está dada como el producto presión - tiempo durante la inspiración. La aplicación de un soporte ventilatorio en la inspiración está encaminada a facilitar el esfuerzo inspiratorio, disminuir el consumo de oxígeno $\left(\mathrm{O}_{2}\right)$ y la producción de dióxido de carbono $\left(\mathrm{CO}_{2}\right)$ garantizando volúmenes minuto adecuados (VE).

\section{Difusión}

En la etapa final de la respiración el aporte de oxígeno a nivel de la circulación pulmonar y la remoción del dióxido de carbono, dependen de la difusión de estas moléculas a través de la membrana alvéolo-capilar. La efectividad de este proceso depende de tres variables:

- Área de difusión existente entre los dos compartimentos (aire - sangre).

- Diferencia entre presiones parciales de las moléculas entre los compartimentos.

- Grado de ventilación - perfusión (cortocicuito) existente.

La manipulación de estas variables garantiza y facilita el aporte y la remoción adecuada a los requerimientos del individuo (14).

\section{Efectos de la ventilación mecánica no invasiva}

\section{Eficacia sobre el trabajo respiratorio}

La definición de trabajo ya se ha valorado previamente y el soporte ventilatorio está orientado a facilitar el esfuerzo inspiratorio con sus consecuencias respectivas. En la actualidad la mayoría de los efectos de la VMNI sobre el trabajo respiratorio se han realizado en diferentes grupos de pacientes: enfermedad pulmonar crónica (EPOC) (15), enfermedad pulmonar restrictiva (16), falla respiratoria hipoxémica aguda, síndrome de hipoventilación (17), obesidad (18), edema pulmonar agudo cardiogénico (19), distrofia muscular de Duchenne, enfermedades neuromusculares (20) y lesión pulmonar aguda (ALI) (21). Su efectividad se ha valorado a través de diferentes parámetros como electromiografía diafragmática (EMGdi), consumo de oxígeno $\left(\mathrm{VO}_{2}\right)$, gasto energético en reposo, tolerancia al ejercicio y disnea. Estos datos revelan que el trabajo respiratorio es mayor en pacientes con algunas de estas patologías, dado por un incremento en las deflecciones negativas en las medidas de presiones esofágica $(\Delta \mathrm{Pes})$ y transdiafragmática $(\mathrm{Pdi})$, las cuales alcanzan $14-16 \mathrm{~cm} \mathrm{H}_{2} \mathrm{O}$. Si se realiza una correlación de la curva de presión - tiempo con el consumo de oxígeno se alcanzan valores hasta $350 \mathrm{~cm} \mathrm{H}_{2} \mathrm{O}$ s/ min, mayores de lo usual. La medición del trabajo (en unidades joules) muestra que el gasto normal es cercano a $1,23 \mathrm{~J} / \mathrm{L}$, mientras este grupo de pacientes genera valores hasta de $13,7 \mathrm{~J} / \mathrm{min}$. Los valores de PEEP intrínseca dinámica (PEEPi) deben estar por debajo de los valores de presión de los músculos inspiratorios para iniciar el flujo de aire inspiratorio; estas medidas son superadas en $3 \mathrm{~cm} \mathrm{H}_{2} \mathrm{O}$ o $5 \mathrm{~cm}$ $\mathrm{H}_{2} \mathrm{O}$ en pacientes críticamente enfermos. La VMNI genera eficacia respecto al trabajo ya que conduce a una reducción ( $\triangle \mathrm{Pes})$ de $8-15 \mathrm{~cm} \mathrm{H}_{2} \mathrm{O}(50-76 \%)$, reduce la Pdi de 5-10 $\mathrm{cm} \mathrm{H}_{2} \mathrm{O}$ (42-62\%) (20). La reducción en PTP oscila entre $127 \mathrm{~cm} \mathrm{H}_{2} \mathrm{O}$ s/min a 345 $\mathrm{cm} \mathrm{H}_{2} \mathrm{O} \mathrm{s} / \mathrm{min}$, lo que representa una disminución de $20 \%$ al $78 \%$, en promedio $189 \mathrm{~cm} \mathrm{H}_{2} \mathrm{O} \mathrm{s} / \mathrm{min}$ (55\%). La medida en unidades joules para valorar la reducción del trabajo respiratorio mostró disminución con la VMNI desde 0,27 a 1,3 J/L (31-69\%) (21-22). La disminución en la percepción de disnea estuvo entre 
29 y $67 \%$ medida de esfuerzo inspiratorio y trabajo. La VMNI causó reducciones sustanciales en las medidas de la electromiografía diafragmática (EMGdi), que van del $17 \%$ al $93 \%$, que significa reducción en el esfuerzo inspiratorio. En general todas las medidas de trabajo se reducen en aproximadamente el $60 \%$ con la VMNI. El tiempo de reducción en los esfuerzos inspiratorios y de la actividad de la (EMGdi), se logra durante 5 a 6 respiraciones.

\section{Efectos sobre la resistencia y la fuerza muscular}

En la mayoría de estas patologías la distensibilidad del parénquima pulmonar disminuye, mientras aumenta la resistencia de las vía aéreas. El aumento de la resistencia podría deberse al menor volumen pulmonar, a la broncoconstricción mediada por eferentes vagales; al estrechamiento de la vía aérea pequeña por aumento de la presión hidrostática en el espacio intersticial broncovascular o por la compresión de las vías aéreas centrales. La mayor resistencia de la vía aérea puede provocar limitación al flujo espiratorio (LFE), generando PEEP intrínseca dinámica (PEEPi) y como consecuencia de estos fenómenos, aumento de la resistencia de las vías aéreas y del WOB (trabajo muscular). La aplicación de una presión positiva en la vía aérea origina un gradiente de presión que da lugar a mejoría en el flujo inspiratorio realizándose la espiración de forma pasiva nuevamente, debido a la retracción elástica pulmonar. Para cada gradiente de presión, el flujo generado dependerá de la resistencia de las vías aéreas y del cambio de volumen alveolar. En algunos estudios se ha correlacionado la tolerancia al ejercicio como una medida indirecta de la función de los músculos inspiratorios y de la resistencia de las vías aéreas encontrando un incremento entre $14-95 \%$ en este grupo de pacientes (23). Con el uso de la VMNI se logra una disminución en las resistencias de las vías aéreas en promedio de 37\% (11 $\left.\mathrm{cm} \mathrm{H}_{2} \mathrm{O}\right)(24)$.

\section{Efectos relativos del nivel de presión soporte}

El determinar la eficacia de la VMNI se requiere diferenciar los efectos del CPAP y los del soporte inspiratorio. La presión inspiratoria positiva reduce el WOB por disminucion del producto presión
- tiempo (PTP) correlacionado con el consumo de oxígeno, durante la inspiración o efecto de "pushpull". Este mecanismo es diferente al PEEP que reduce el WOB por dos acciones: por contrapeso sobre el PEEPi y de este modo por reducción del umbral de carga inspiratoria (25), y por incremento de la distensibilidad del sistema respiratorio y así por reducción de la carga elástica en la inspiración (25). Diferentes estudios han comparado los efectos del CPAP con los del soporte de la inspiración más PEEP sobre el trabajo respiratorio y la disnea. En los pacientes con EPOC y con hipercapnia crónica, Nava (26) encontró que el incremento en la presión soporte de $10 \mathrm{~cm} \mathrm{H}_{2} \mathrm{O}$ a $20 \mathrm{~cm} \mathrm{H}_{2} \mathrm{O}$ causa

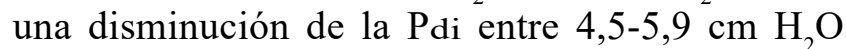
(35-46\%) y adicionalmente reduce PTP en 50-65\%. Al aplicar PEEP de $5 \mathrm{~cm} \mathrm{H}_{2} \mathrm{O}$ se tendrá un efecto adicional y se reducirá significativamente la EMGdi. En pacientes con EPOC la aplicación de presión soporte paso a paso desde 5 hasta $20 \mathrm{~cm} \mathrm{H}_{2} \mathrm{O}$ de forma progresiva, también redujo WOB y la Pdi en 15 a $20 \%$ en cada paso. Mientras se mantiene una presión inspiratoria constante, adicionando el PEEP de $5 \mathrm{~cm} \mathrm{H}_{2} \mathrm{O}$ a $10 \mathrm{~cm} \mathrm{H}_{2} \mathrm{O}$, generalmente se produce un aumento en la Pdi que lleva al mismo nivel de la presión inspiratoria pico que si no tuviera PEEP. Sin embargo, $10 \mathrm{~cm} \mathrm{H}_{2} \mathrm{O}$ fue menos efectivo en reducir el trabajo de los músculos inspiratorios que cuando se usó una presión soporte con un nivel de 15-20 $\mathrm{cm} \mathrm{H}_{2} \mathrm{O}$ con o sin PEEP. Hallazgos similares encontraron Appendini, que al combinar PEEP de $5 \mathrm{~cm} \mathrm{H} \mathrm{H}_{2} \mathrm{O}$ con presión soporte de $10 \mathrm{~cm} \mathrm{H}_{2} \mathrm{O}$ redujeron la PTPdi más (229 $\left.\mathrm{cm} \mathrm{H}_{2} \mathrm{O} \mathrm{s} / \mathrm{min}, 53 \%\right)$ que con presión soporte de $10 \mathrm{~cm} \mathrm{H}_{2} \mathrm{O}\left(110 \mathrm{~cm} \mathrm{H} \mathrm{H}_{2} \mathrm{O}\right.$ s/min, 22\%) o CPAP de $5 \mathrm{~cm} \mathrm{H}_{2} \mathrm{O}\left(83 \mathrm{~cm} \mathrm{H}_{2} \mathrm{O}\right.$ s/ min, $19 \%$ ) en pacientes críticamente enfermos con EPOC. Se ha encontrado que la ventilación proporcional asistida (PAV) con PEEP adicional de 5 $\mathrm{cm} \mathrm{H}_{2} \mathrm{O}$ mejora la tolerancia al ejercicio $(26 \%)$ o PAV sin PEEP (8\%). La ventilación no invasiva a través de CPAP sola ,reduce el trabajo inspiratorio y la carga de trabajo en los pacientes con EPOC. Además la aplicación paso a paso de presión con CPAP de $10 \mathrm{~cm} \mathrm{H}_{2} \mathrm{O}$ causa una reducción progresiva en el PEEPi de aproximadamente 69\%, PTP y la Pdi $40-48 \%$, respectivamente, lo que conduce a 
una mejoría en 1,1 L en los volúmenes al final de la espiración. El PEEP ajustado logra la comodidad del paciente y la mejoría fisiológica conduciendo a reducción de trabajo inspiratorio a $29-20 \%$ asociado a control del PEEPi dinámico.

En los pacientes con lesión pulmonar aguda se ha reportado que el uso de CPAP de $10 \mathrm{~cm} \mathrm{H}_{2} \mathrm{O}$ reduce la PTP en $16 \%$ (40 cm H 20 s/min), en contraste con la presión soporte que reduce la PTP en 50\% (133-142 cm $\mathrm{H}_{2} \mathrm{O} \mathrm{s} / \mathrm{min}$ ). También han reportado que el uso de BiPAP con presión soporte de $5 \mathrm{~cm}$ $\mathrm{H}_{2} \mathrm{O}$ mejora el PEEP y la disnea más que el uso de CPAP con presión de $10 \mathrm{~cm} \mathrm{H}_{2} \mathrm{O}$ (27).

\section{Efectos relativos de los modos de la ventilación mecánica no invasiva}

Varios estudios han comparado la efectividad de diferentes modos para reducir el trabajo respiratorio durante la VMNI (PAV vs. PSV). En los pacientes con EPOC se encontraron reducciones similares en la disnea $30 \%$ y en el incremento de la resistencia al ejercicio. Entre pacientes hospitalizados con fibrosis quística (PSV y PAV), ambos modos logran el mismo nivel de apoyo inspiratorio y conducen a una reducción similar en la EMGdi 14-17\% respectivamente (28). En cuanto a la disnea y la calidad del sueño en pacientes con EPOC y enfermedades restrictivas, la PSV y PAV lograron los mismos niveles de presión inspiratoria pico y PEEP, aunque la PAV presentó menor efecto de boca o nariz secas y mayor confort, lo cual se ha relacionado con un incremento y variabilidad del Vt que responde de forma continua a las demandas dadas por el esfuerzo y la mecánica pulmonar del paciente (28). En otros estudios se han comparado la ventilación controlada por volumen (VCV) con BiPAP en pacientes con EPOC estable. Ambos modos fueron iguales en cuanto al confort del paciente. Entre dichos modos se observó disminución de la $\Delta$ Pes de 9,5 cm $\mathrm{H}_{2} \mathrm{O}$ vs. 8,8 $\mathrm{cm} \mathrm{H}_{2} \mathrm{O}$, respectivamente, comparado con el modo no asistido, con el cual se logró una reducción de la respiración espontánea en $90 \%$. En los pacientes con EPOC y con falla respiratoria aguda hipoxémica, se ha reportado disminución en el trabajo respiratorio y PTP en el modo VCV más que en el PSV.

\section{Confort del paciente frente a la optimización de la función muscular respiratoria}

Algunos estudios muestran la mayor reducción del WOB inspiratorio con el VCV, pero paradójicamente el disconfort con este modo es mayor que con el PSV. El modo PSV conduce a una disminución

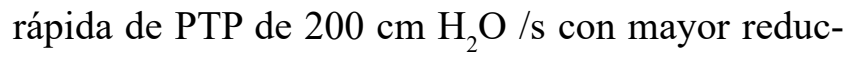
ción de Pdi $62 \%$ y de $\Delta$ Pes $54 \%$, pero también pobre tolerancia y mayor presencia de fugas en el circuito. Algunos estudios tienen combinaciones entre incrementos de la presión soporte y el PEEP para producir una mejoría lineal sobre el drive respiratorio y el patrón respiratorio. No obstante, el confort de los pacientes presenta una curva en forma de $\mathrm{U}$, en donde se observa mayor incomodidad con niveles bajos o altos de soporte de presión. La zona de máximo confort ocurre entre un valor de PEEP de $5 \mathrm{~cm} \mathrm{H}_{2} \mathrm{O}$ con una presión soporte de $5-10 \mathrm{~cm} \mathrm{H}_{2} \mathrm{O}$ o con PEEP de cero y presión soporte de $15 \mathrm{~cm} \mathrm{H}_{2} \mathrm{O}$. La disminución del confort con presiones altas se explica por la falta de coordinación entre paciente - ventilador y la presencia de esfuerzos no capturados (29).

\section{Efectos de las interfases}

Se han comparado los efectos de diferentes interfases (máscaras nasales, almohadillas nasales, oronasales y faciales) respecto al patrón y la tolerancia de la VMNI; se conoce que, a pesar de lograr un mejor $\mathrm{Vt}$ y un flujo pico máximo con una máscara facial, los pacientes se inclinan por las máscaras nasales. Una complicación importante asociada con la VMNI a largo plazo es la ruptura de la piel y la pérdida de confort, que generan el 18\% de los fracasos asociados con su uso. Un estudio en sujetos normales no encontró diferencias significativas en cuanto al WOB con el uso de máscaras grandes o pequeñas, pero otros han mostrado que el WOB durante la PSV con máscara facial se reduce a cero en un tiempo promedio para alcanzar el nivel de presión de soporte de $330 \mathrm{~ms}$, mientras que con el Helmet el tiempo para alcanzar el nivel de presión de soporte estaba entre $1,020 \mathrm{~ms}$ y $960 \mathrm{~ms}$ (30). Estos resultados sugieren precaución cuando se considere una interfase Helmet, particularmente en quienes tienen hipercapnia severa y cuando se requiere un incremento rápido en la ventilación alveolar. 


\section{Dispositivos de humidificación}

A pesar de que la vía aérea superior no se interviene de manera directa durante la VMNI, la entrega prolongada de gas seco a alto flujo, puede ser superior a la capacidad de estas estructuras anatómicas para proporcionar humidificación adecuada; por tanto, se recomienda la humidificación suplementaria durante la VMNI. La elección del dispositivo puede generar impacto sobre el trabajo respiratorio, por ejemplo: un intercambiador de calor y humedad, en comparación con un humidificador térmico, durante la VMNI se asocia con mayor WOB $(0,66 \mathrm{~J} / \mathrm{L}$ vs. $0,36 \mathrm{~J} / \mathrm{L}$ ) y mayor trabajo muscular (W: $15,5 \mathrm{~J} / \mathrm{min}$ frente a $8,4 \mathrm{~J} / \mathrm{min}$ )

\section{Patrón de la respiración}

Se han evaluado los cambios en el patrón de la respiración. Las presiones inspiratorias conducen a reducciones sobre la carga inspiratoria con un aumento en el Vt aproximadamente de $230 \mathrm{ml}(47 \%)$ y algunos cambios variables en la frecuencia respiratoria. En la mayoría de los estudios se produjo una disminución de 6 respiraciones/min (22\%). A pesar de la disminución en la frecuencia respiratoria el aumento del $\mathrm{Vt}$ conduce a un aumento de la ventilación minuto ( $\left.\dot{\mathrm{V}}_{\mathrm{E}}\right)$ en $3 \mathrm{~L} / \mathrm{min}(31 \%)(31)$.

\section{Efectos fisiológicos en la enfermedad pulmonar obstructiva crónica}

Una de las alteraciones fisiopatológicas centrales de la EPOC es la limitación del flujo espiratorio (LFE); las exacerbaciones de la EPOC empeoran bruscamente la LFE, debido a inflamación y broncoconstricción de las vías aéreas, lo cual conduce a hiperinflación dinámica, aumento del trabajo respiratorio, alteraciones del intercambio gaseoso y disfunción de la musculatura respiratoria. El tiempo espiratorio insuficiente aumenta el volumen al final de la espiración (EELV) y genera hiperinflación pulmonar dinámica (HD).

Además el patrón respiratorio generado por una carga inspiratoria elevada es rápido y superficial, limitando mucho más el tiempo espiratorio disponible para el vaciamiento pulmonar, hecho que causa mayor HD y conduce a presiones positivas intrínsecas al final de la espiración (PEEPi o auto-PEEP). La PEEPi actúa como un umbral de carga inspiratoria sobre los músculos inspiratorios que deben vencerla para iniciar el flujo inspiratorio. Los valores de PEEPi oscilan entre 6-9 $\mathrm{cm} \mathrm{H}_{2} \mathrm{O}$ en EPOC estable pero aumenta en exacerbaciones entre $13-20 \mathrm{~cm}$ $\mathrm{H}_{2} \mathrm{O}$, generándose así un incremento del trabajo inspiratorio en $60 \%$.

De otro lado, a diferencia de un sujeto normal que respira en la porción inclinada de la curva presión-volumen, durante una exacerbación debido a la HD, el paciente se ubica en la porción más elevada y plana de la curva, cerca a la capacidad pulmonar total (CPT), en cuyo sitio se necesitan grandes presiones inspiratorias para generar pequeños cambios de volumen. Los músculos respiratorios se adaptan a la hiperinflación crónica durante la respiración tranquila, pero durante el ejercicio esta capacidad es desbordada y causa aumento de la carga inspiratoria, mas conduce a disnea, aumento del WOB y W, alteraciones del intercambio gaseoso como hipoxemia e hipercapnia que refleja la incapacidad en el aumento en el Vt y la no optimización del espacio muerto fisiológico.

La VMNI, con PSV y PEEP, asiste la inspiración y reduce el trabajo respiratorio. Este efecto se debe a que la PEEP contrarresta el efecto de la PEEPi, disminuyendo el trabajo elástico estático, mientras que la PSV reduce el trabajo elástico y resistivo dinámico, que conduce a una disminución de la $\Delta \mathrm{Pes}, \mathrm{Pdi}$, PTP, W y WOB. La reducción del esfuerzo muscular se acompaña de un aumento del VT y una reducción de la frecuencia respiratoria, incrementándose el $\dot{V}$ E., además de mejorar los parámetros de gases arteriales en las primeras horas, como se resalta en otro apartado de este artículo.

\section{Función cardiovascular y edema agudo de pulmón de orígen cardiogénico}

La principal característica del edema pulmonar agudo cardiogénico (EPA) es un incremento rápido de la presión hidrostática de los capilares pulmonares, que aumenta la tasa de filtración transvascular de los fluidos hacia el intersticio y los espacios alveolares. En esta situación la distensibilidad del 
parénquima pulmonar se disminuye y aumenta la resistencia de las vías aéreas. La mayor resistencia de las vías aéreas conduce a limitación al flujo espiratorio (LFE) y generación de PEEPi. La consecuencia de estos fenómenos es el aumento del WOB y de la disnea.

En el curso del EPA los músculos respiratorios generan grandes deflexiones negativas de la presión pleural para iniciar la inspiración y mantener un $\mathrm{Vt}$ adecuado, pero estas presiones negativas agravan el edema, ya que incrementan tanto la precarga como la postcarga ventricular. Esto explica porqué el llenado y vaciado cardíaco son determinados por la diferencia de las presiones intracavitaria e intratorácica, que corresponde a la presión transmural (РтM). La amplitud de las deflexiones inspiratorias es mayor de lo normal; en EPA la PTM se encuentra aumentada, y cuanto mayor es la РTM durante la diástole, mayor es el llenado cardíaco (precarga). A su vez, a mayor PтM durante la sístole, mayor trabajo cardíaco (poscarga).

La aplicación de presión positiva continua de la vía aérea a través de CPAP o de VMNI con PSV y PEEP, conduce a dos tipos de efectos benéficos en el EPA, mejorando la función respiratoria y la función cardíaca.

En efecto, al elevar la presión intratorácica, con CPAP o con VMNI con PSV y PEEP se disminuye el cortocircuito intrapulmonar, optimizando la oxigenación y reduciendo la disnea. Además, el CPAP disminuye las deflexiones inspiratorias durante la sístole y en consecuencia la PTM, generando un descenso en el retorno venoso, y disminuyendo la precarga de ambos ventrículos. Durante la diástole, el CPAP aumenta la presión intratorácica y pericárdica, lo que produce una disminución de la РтM y una reducción de la poscarga. La reducción de la precarga genera una disminución rápida del edema, y la disminución en la pre y poscarga mejoran la función ventricular y pueden promover un aumento del gasto cardíaco. Se ha observado que los efectos sobre la precarga se producen en pacientes con disfunción sistólica y diastólica, diferente al incremento del gasto cardíaco que sólo ocurre cuando está comprometida la contractilidad (33).
Los efectos hemodinámicos de la VMNI son muy variados y también dependen del uso del PEEP, del modo de VMNI. Se han comparado el uso de CPAP de $10 \mathrm{~cm} \mathrm{H}_{2} \mathrm{O}$ con el BiPAP de $15 / 10 \mathrm{~cm} \mathrm{H}_{2} \mathrm{O}$ en este grupo de pacientes y se ha observado una disminución del $19 \%$ del gasto cardíaco $\left(1,1 \mathrm{~L} / \mathrm{min} / \mathrm{m}^{2}\right)$ en enfermos, frente a sujetos normales donde la disminución del gasto cardíaco se percibió de forma significativa en el $31 \%\left(2,3 \mathrm{~L} / \mathrm{min} / \mathrm{m}^{2}\right)$ con presión de 20 $\mathrm{cm} \mathrm{H}_{2} \mathrm{O}$, pero estos hallazgos también se relacionan principalmente con el tipo de interfase utilizada durante la ventilación (34).

Las mejorías en el patrón respiratorio y los gases arteriales ( $\mathrm{pH}$ arterial 7,18-7,28 y $\mathrm{PaCO}_{2} 12 \mathrm{~mm} \mathrm{Hg}$, $21 \%$ ) (35), se han reportado en pacientes con edema pulmonar cardiogénico secundario a infarto agudo de miocardio con VMNI a través del modo PSV cuando se usan presiones inspiratorias y espiratorias de 15-21/4-5 $\mathrm{cm} \mathrm{H}_{2} \mathrm{O}$. En un estudio multicéntrico aleatorizado controlado que comparó $\operatorname{BiPAP}(12,05$ $\left.\mathrm{cm} \mathrm{H}_{2} \mathrm{O}\right)$ con CPAP $\left(8 \mathrm{~cm} \mathrm{H} \mathrm{H}_{2} \mathrm{O}\right)$, los dos modos fueron igual de eficaces en la reducción de la frecuencia cardíaca en 9-22 latidos/min (8-19\%), disnea y en la mejoría los gases arteriales (35-37).

\section{Función en el intercambio gaseoso - pulmonar}

Muchos estudios han mostrado los efectos a corto y largo plazo de la VMNI en el intercambio gaseoso en pacientes con enfermedad pulmonar. Algunos de estos, a largo plazo (días o meses) en pacientes que recibieron VMNI domiciliaria, concordaron en que la tendencia de los hallazgos era hacia la mejoría. En general, se produce aumento del $\mathrm{pH}$ arterial en 0,06 , disminución de la $\mathrm{PaCO}_{2}$ en promedio de $9 \mathrm{~mm} \mathrm{Hg}$, elevación de la $\mathrm{PaO}_{2}$ en $8 \mathrm{~mm} \mathrm{Hg}$ y mejoría en la relación de $\mathrm{PaO}_{2}$ y la fracción de oxígeno inspirado $\left(\mathrm{PaO}_{2} / \mathrm{FiO}_{2}\right)$ que se incrementó en $27 \mathrm{~mm} \mathrm{Hg}(34)$.

\section{Conclusiones}

Este artículo muestra los efectos fisiológicos de la VMNI con resultados destacados como la reducción en el promedio del WOB que es $15 \mathrm{~cm} \mathrm{H}_{2} \mathrm{O}$ y que está relacionado con los cambios en $\Delta$ Pes y en Pdi durante la ventilación, aumento del Vt que 
conduce a mejoría del $\dot{V}_{E}$, capacidad del PEEP aplicado para contrarrestar la sobrecarga de los efectos de PEEPi, reducción en la percepción de la disnea, resincronización del drive respiratorio y mejoría de los parámetros gasimétricos.

La reducción del WOB se logra con una presión soporte de $15 \mathrm{~cm} \mathrm{H}_{2} \mathrm{O}$ y un PEEP de $5 \mathrm{~cm} \mathrm{H}_{2} \mathrm{O}$ y retorno del esfuerzo inspiratorio a los niveles normales, principalmente en pacientes con enfermedad pulmonar crónica. También se establece que no se puede lograr un reposo absoluto de los músculos inspiratorios dado que las presiones de $20 \mathrm{~cm} \mathrm{H}_{2} \mathrm{O}$, conducen a mayor número de fugas y menor confort. Por lo tanto, la posibilidad de una descarga completa de los músculos inspiratorios del paciente se ve reprimida por el límite superior de la presión inspiratoria positiva. Todas estas variables deben considerarse en el momento de iniciar este tipo de soporte.

\section{Bibliografía}

1. Barach AL, Martin J, Eckman M. Positive pressure respiration and its application to the treatment of acute pulmonary edema. Ann Intern Med. 1938;12:754-95.

2. Alba A, Khan A, Lee M. Mouth IPPV for sleep. Rehabilitation gazette. 1984;24:47-9.

3. Bach JR, Alba AS. Management of chronic alveolar hypoventilation by nasal ventilation. Chest. 1990;97:52-72.

4. ThokalaP, Goodacre S, sala M, Penn-Ashman J. Cost-effectiveness of out-of-hospital continuous positive airway pressure for acute respiratory failure. Ann Emerg Med. 2015:196-204.

5. Hedly-Whyte J, Burgess GE, Feeley TW, Miller MG. Applied physiology of respiratory care. Chapter 2: Effect of controlled ventilation on the lungs and cardiovascular system. Boston: Little, Brown and Company; 1976. p. 13-35.

6. Suter PM, Fairley HB, Isenberg MD. Optimum end-expiratory airway pressure in patients with acute pulmonary failure. $\mathrm{N}$ Engl J Med. 1975;292(6):284-9.

7. Kallet RH, Campbell AR, Dicker RA. The effects of tidal volume demand on work of breathing during lung protective ventilation in patients with acute lung injury and acute respiratory distress syndrome. Crit Care Med. 2006;34(1):8-14.

8. Flick GR, Bellamy PE. Diaphragmatic contraction during assisted mechanical ventilation. Chest. 1989;96(1):130-5.

9. Colgan FJ, Barrow RE, Fanning GL. Constant positive-pressure breathing and cardiorespiratory function. Anesthesiology. 1971;34:145-51.

10. Morgan BC, Martin WE, Hornbein TF, Crawford EW. Hemodynamic effects of intermittent positive pressure respiration. Anesthesiology. 1966;27(5):584-90.
11. Dreyfuss D, Soler P, Basset G. Respective effects of high airway pressure, high tidal volumen and positive end expiratory pressure. Am Rev Resp Dis. 1988;137:1159-64.

12. Shaffer TH, Wolfson MR, Panitch HB. Airway structure, function and development in health and disease. Paediart Anaesth. 2004;14:3-14.

13. Ferris Bg, Mead J, Opie LH. Partitioning of respiratory flow resistance in man. J Appl Physiol. 1964;19:653-8.

14. Bryan AC, Bentiglovio LG, Beerel F. Factors affecting regional distribution of ventilation and perfusion in the lung. J Appl Physiol .1964; 19: 395-402.

15. Carrey Z, Gottfried SB, Levy RD. Ventilatory muscle support in respiratory failure with nasal positive pressure ventilation. Chest. 1990;97(1):150-8.

16. Winck JC, Vitacca M, Morais A, Barbano L, Porta R, TeixeiraPinto A, Ambrosino N. Tolerance and physiologic effects of nocturnal mask pressure support vs. proportional assist ventilation in chronic ventilatory failure. Chest. 2004;126(2):3828.

17. Girault C, Richard J-C, Chevron V, Tamion F, Pasquis P, Leroy J, Bonmarchand G. Comparative physiologic effects of noninvasive assist-control and pressure support ventilation in acute hypercapnic respiratory failure. Chest. 1997;111(6):1639-48.

18. Waldhorn RE. Nocturnal nasal intermittent positive pressure ventilation with bi-level positive airway pressure (BiPAP) in respiratory failure. Chest. 1992;101(2):516-21.

19. Kramer N, Meyer TJ, Meharg J, Cece RD, Hill NS. Randomized prospective trial of noninvasive positive pressure ventilation in acute respiratory failure. Am J Respir Crit Care Med. 1995;151(6):1799-806.

20. Toussaint M, Soudan P, Kinnear W. Effect of noninvasive ventilation on respiratory muscle loading and endurance in Duchenne patients. Thorax. 2008;63(5):430-4.

21. L'Her E, Deye N, Lellouche F, Taille S, Demoule A, Fraticelli A, et al. Physiologic effects of noninvasive ventilation during acute lung injury. Am J Respir Crit Care Med. 2005;172(9):1112-8.

22. deLucas P, Tarancon C, Puente L, Rodriguez C, Tatay E, Monturiol JM. Nasal continuous positive airway pressure in patients with COPD in acute respiratory failure. Chest. 1993;104(6):1694-7.

23. Renston JP, DiMarco AF, Supinski GS. Respiratory muscle rest using nasal BiPAP ventilation in patients with stable severe COPD. Chest .1994;105(4):1053-60.

24. Katz JA, Marks JD. Inspiratory work with and without continuous positive airway pressure in patients with acute respiratory failure. Anesthesiology. 1985;63(6):598-607.

25. Sydow M, Golish W, Buscher H, Zinserling J, Crozier TA, Burchardi H. Effect of low-level PEEP on inspiratory work of breathing in intubated patients, both with healthy lungs and with COPD. Intensive Care Med 1995;21(11):887-95. 
26. Nava S, Ambrosino N, Rubini F, Fracchia C, Rampulla C, Torri G, Calderini E. Effect of nasal pressure support ventilation and external PEEP on diaphragmatic activity in patients with severe stable COPD. Chest. 1993;103(1):143-50.

27. Mehta S, Jay GD, Woolard RH, Hipona RA, Connolly EM, Cimini DM, Drinkwater JH, Hill NS. Randomized prospective trial of bilevel versus continuous positive airway pressure in acute pulmonary edema. Crit Care Med. 1997;25(4):620-8.

28. Wysocki M, Richard JC, Meshaka P. Noninvasive proportional assist ventilation compared with noninvasive pressure support ventilation in hypercapnic acute respiratory failure. Crit Care Med 2002;30(2):323-9.

29. Nava S, Bruschi C, Rubini F, Palo A, Iotti G, Braschi A. Respiratory response and inspiratory effort during pressure support ventilation in COPD patients. Intensive Care Med. 1995;21(11):871-9.

30. Racca F, Appendini L, Gregoretti C. Effectiveness of mask and helmet interfaces to deliver noninvasive ventilation in a human model of resistive breathing. J Appl Physiol. 2005;99(4):1262-71.

31. Ambrosino N, Nava S, Bertone P, Fracchia C, Rampulla C. Physiologic evaluation of pressure support ventilation by nasal mask in patients with COPD. Chest. 1992;101(2):385-91.

32. Porta R, Vitacca M, Clini E, Ambrosino N. Physiological effects of posture on mask ventilation in awake sta- ble chronic hypercapnic COPD patients. Eur Resp J. 1999;14(3):517-22.

33. Kilger E, Briegel J, Haller M, Frey L, et al. Effects of noninvasive positive pressure ventilatory support in non- COPD patients with acute respiratory insufficiency after early extubation. Intensive Care Med. 1999;25(12):1374-80.

34. Ambrosino N, Nava S, Torbiki A, Riccardi G, Fracchia C, Opasich C, Rampulla C. Haemodynamic effects of pressure support and PEEP ventilation by nasal route in patients with stable chronic obstructive pulmonary disease. Thorax. 1993;48(5):523-8.

35. Bersten AD, Holt AW, Vedig AE, Skowronski GA, Baggoley CJ. Treatment of severe cardiogenic pulmonary edema with continuous positive airway pressure delivered by face mask. N Engl J Med. 1991;325(26):1825-30.

36. Rasanen J, Heikkila J, Downs J, Nikki P, Vaisanen I, Viitanen A. Continuous positive airway pressure by face mask in acute cardiogenic pulmonary edema. Am J Cardiol. 1985;55(4):296-300.

37. Masip J, Betbese AJ, Paez J, et al. Noninvasive pressure support ventilation versus conventional oxygen therapy in acute cardiogenic pulmonary oedema: a randomized trial. Lancet. 2000;356(9248):2126-32. 